Research Article

\title{
A SNP in 5' untranslated region of CD40 gene is associated with an increased risk of ischemic stroke in a Chinese population: a case-control study
}

\author{
Hua-Tuo Huang ${ }^{1}$, Jing Guo ${ }^{3}$, Yang Xiang ${ }^{1}$, Jian-Ming Chen ${ }^{1}$ Hong-Cheng Luo ${ }^{1}$, Lan-Qing Meng ${ }^{2}$ and \\ Ye-Sheng $\mathrm{Wei}^{1}$ \\ ${ }^{1}$ Department of Laboratory Medicine, Affiliated Hospital of Youjiang Medical University for Nationalities, \\ Baise, Guangxi, China. \\ ${ }^{2}$ Department of Neurology, Affiliated Hospital of Youjiang Medical University for Nationalities, Baise, \\ Guangxi, China. \\ ${ }^{3}$ Department of Dermatology, Affiliated Hospital of Youjiang Medical University for Nationalities, Baise, \\ Guangxi, China.
}

\begin{abstract}
Cluster of differentiation 40 (CD40), the receptor for CD154, is a member of the tumor necrosis factor (TNF) receptor superfamily. Several studies have been conducted to investigate the effect of the CD40 rs 1883832 polymorphism on atherosclerotic disease in different population; however, inconsistent results were obtained. In this study, we investigated the association of four polymorphisms (rs1883832, rs13040307, rs752118 and rs3765459) of CD40 gene and their effect on CD40 expression with the risk of ischemic stroke (IS) in a Chinese population. Three hundred and eighty patients with IS and 450 control subjects were included in the study. The CD40 polymorphisms were discriminated by Snapshot SNP genotyping assay. Serum soluble CD40 (sCD40) levels were detected by ELISA. We found that the rs $1883832 \mathrm{CT}$ and rs1883832TT genotypes were associated with an increased risk of IS compared with the rs $1883832 C$ Cenotype $(\mathrm{OR}=1.42,95 \% \mathrm{Cl}: 1.03-1.95, \mathrm{p}=0.030$ and $\mathrm{OR}=1.91,95 \% \mathrm{Cl}: 1.29-2.82, P=0.001$, respectively), and the rs $1883832 \mathrm{~T}$ allele was associated with a significantly increased risk of IS compared with rs1883832C allele ( $\mathrm{OR}=1.40,95 \% \mathrm{Cl}: 1.15-1.70, P=0.001)$. Elevated serum sCD40 levels were observed in patients with IS compared with the control gropu $(P<0.01)$. Individuals carrying the rs $1883832 \mathrm{TT}$ or rs $1883832 \mathrm{CT}$ genotypes showed significantly higher SCD40 levels compared with the rs1883832CC genotype in the IS group [ $64.8 \pm$ $25.4 \mathrm{pg} / \mathrm{mL}, \mathrm{TT}=94) ;(63.9 \pm 24.3 \mathrm{pg} / \mathrm{mL}, \mathrm{CT}=185) \mathrm{vs}(53.3 \pm 22.5 \mathrm{pg} / \mathrm{mL}, \mathrm{CC}=101), P<0.01]$. The TCCA haplotype was associated with an increased risk of IS compared with the control group $(\mathrm{OR}=2.10,95 \% \mathrm{Cl}$ : $1.23-3.58, p=0.005)$. However, we did not find a significant association between the other three polymorphisms and IS risk. In conclusion, after a comprehensive comparison with other studies, we confirmed that the rs1883832T allele but not the rs $1883832 \mathrm{C}$ allele is associated with an increased risk of IS. The rs1883832 polymorphism may exert influences on abnormal CD40 expression in IS patients among the Chinese population.
\end{abstract}

Keywords: CD40, gene, polymorphism, ischemic stroke.

Received: September 06, 2016; Accepted: January 04, 2017.

\section{Introduction}

Stroke is one of the leading causes of death and a common cause of long-term disability in the world (Feigin et al., 2009, Lloyd-Jones et al., 2010). In China, there are approximately 2.5 million new strokes, and more than one million people die from stroke-related causes every year (Liu et al., 2011). Ischemic stroke (IS) is the most common

Send correspondence to Ye-Sheng Wei. Department of Laboratory Medicine, Affiliated Hospital of Youjiang Medical University for Nationalities, Baise 533000, Guangxi, China. E-mail: hhtzyy99@126.com type of stroke, accounting for more than $80 \%$ of cases (Liu et al., 2011). Previous studies have identified that age and sex are closely related to IS (Lloyd-Jones et al., 2010; Turtzo and McCullough, 2010). Hypertension, smoking, alcohol abuse, diabetes mellitus, and hypercholesterolemia were demonstrated as important risk factors for IS (Sacco et al., 1997). However, these risk factors together explain only about $50 \%$ of the risk (Sacco et al., 1989), indicating that other factors such as immune, inflammatory and genetic factors may also be involved in the pathogenesis of IS. In the last few years, candidate genes for IS have been in- 
tensely studied, and numerous susceptible candidates including CD40 gene have been found.

$\mathrm{CD} 40$, the costimulatory receptor for CD40 ligand (CD40L/CD154), is a $48-\mathrm{kDa}$ type I transmembrane protein receptor belonging to the tumor necrosis factor (TNF) receptor superfamily (Elgueta et al., 2009). Overexpression of CD40 and/or its ligand CD40L have been detected in patients with atherosclerosis-related diseases such as coronary artery disease and stroke, and were suggested as potential biomarkers for predicting cardiovascular disease (Yan et al., 2002; Yan et al., 2004; Li et al., 2015). Binding of CD40L to its receptor in vascular endothelial cells triggers the transcription of proinflammatory and proatherogenic genes, which are important components in the onset of atherosclerosis-related diseases, including stroke (Chen et al., 2006). It is accepted that atherosclerosis, plaque instability and thrombus are important pathological basis of IS. In vitro, binding of CD40L to its receptor on the surface of endothelial cells and smooth muscle cells leads to the activation of these cells, resulting in the expression of adhesion molecules, which is an initiating step of atherogenesis (Schonbeck and Libby, 2001; Schonbeck et al., 2002). Moreover, the interaction between CD40 and its ligand induces the expression of matrix metalloproteinases (MMP), resulting in the degradation of interstitial collagen and the thin fibrous cap of atheromatous plaques, and eventually leading to the instability and rupture of plaques (Schonbeck and Libby, 2001). Furthermore, the CD40-CD40L interaction promotes the expression of tissue factors on macrophage cells and endothelial cells, leading to decreased thrombomodulin expression, and favoring a local procoagulant and prothrombotic status (Aukrust et al., 2004). These data indicate that CD40 might play a pathological role in IS, and it may be used as a biomarker and therapeutic target for IS. Hence, the CD40 gene is likely a potential candidate gene for IS risk.

The gene encoding CD40 is located on 20q12-q13.2 in humans, which is consisted of 9 exons and 8 introns. The rs 1883832 locus, previously demonstrated to be associated with CD40 expression, is located at the -1 position within the Kozak sequence (Jacobson et al., 2005; Tian et al., 2010). Several studies have investigated the association between rs 1883832 polymorphism and risk of atherosclerotic disease in different populations, however, the results were inconclusive (Yan et al., 2010; Wang et al., 2011; Ma et al., 2013; Zhang et al., 2013). To confirm the results, we conducted a case-control study with 380 IS patients and 450 control subjects. Moreover, three new polymorphisms (rs13040307, rs752118 and rs3765459) were added in this study. Until now, little information has addressed the effect of CD40 polymorphisms on CD40 expression and their effect on IS risk. The aim of the present study was to investigate the role of these polymorphisms in the genetic basis of IS and to assess the relationship between CD40 polymorphisms and serum level of CD40 in a Chinese population.

\section{Materials and Methods}

\section{Study population}

The study protocol was approved by the ethics committee of Affiliated Hospital of Youjiang Medical University for Nationalities, and informed consent was obtained from all the IS patients and control subjects. The study population included 380 IS patients ( 290 men and 90 women, mean age: $60.7 \pm 13.2$ years) and 450 control subjects ( 325 men and 125 women, mean age: $63.9 \pm 10.3$ years). All IS patients were recruited from the Department of Neurology of the institution from May 2013 to December 2014. The control subjects were frequency-matched with the IS group on the basis of age and sex. All the control subjects were recruited from the physical examination center of the same hospital from July 2013 to December 2013. According to thorough clinical and laboratory evaluation, none of them were found to have any medical condition other than hypertension, diabetes, hypercholest erolemia or hypertriglyceridemia. All participants were Han Chinese and were consecutively selected from the same geographic region of Guangxi, China.

IS patients were classified in accordance with the Trial of Org 10172 in Acute Stroke Treatment (TOAST) classification (Adams Jr et al., 1993) as large-artery atherosclerosis (LAA), small-artery occlusion (SAO), cardioembolism or stroke of other determined etiology. Classifications were based on clinical findings, neuroimaging data [computed tomography (CT) and/or cranial magnetic resonance imaging (MRI)] and results of diagnostic studies such as duplex imaging of extracranial arteries, cardiac imaging (echocardiography) and laboratory evaluation.

Hypertension was diagnosed if the diastolic blood pressure was $\geq 90 \mathrm{mmHg}$ and/or the systolic blood pressure was $\geq 140 \mathrm{mmHg}$, or if the person was currently using antihypertensive treatment. Hypercholesterolemia was defined as fasting serum total cholesterol level $>6.2 \mathrm{mmol} / \mathrm{L}$, and hypertriglyceridemia was diagnosed if the fasting serum triglyceride level was $>2.3 \mathrm{mmol} / \mathrm{L}$.

\section{DNA extraction and genotyping}

Genomic DNA was extracted from venous blood leukocytes by using a salting-out method (John et al., 1991). Genotyping method was described in detail previously (Chen et al., 2015a). Briefly, SnapshotSNPgenotyping assay was used to determine the genotypes of rs1883832, rs13040307, rs752118 and rs3765459 polymorphisms. PCR primers were designed in accordance with the GenBank reference sequence (accession no. NC_000020.11). Moreover, DNA sequencing method was used to confirm our genotyping results.

\section{Serum sCD40 determination}

Serum samples from IS patients and control subjects were separated from venous blood at room temperature and 
stored at $-70{ }^{\circ} \mathrm{C}$ until use. Serum sCD40 levels were analyzed by enzyme linked immunosorbent assay (ELISA) kits (Bender Med Systems, USA) according to the protocol of the manufacturer. Developed color reaction was quantified by an ELISA reader (RT-6000, China). The concentration of serum SCD40 was determined by using a standard curve constructed with the kit's standards over the range of $0-500 \mathrm{pg} / \mathrm{mL}$.

\section{Statistical analysis}

Statistical analyses were performed using the SPSS software program version 17.0. Continuous variables are displayed as mean \pm standard deviation (SD). If the data were normally distributed, the Student's $t$-test was used. Categorical variables are reported as proportions and compared using the chi-square test. Hardy-Weinberg equilibrium (HWE) was tested by the chi-square test. The Shi's standardized coefficient D' (D') (Shi and He, 2005) was used to quantify the linkage disequilibrium (LD) between polymorphisms. Haplotypes and their frequencies were estimated on the basis of a Bayesian algorithm using the Phase program (Stephens et al., 2001). The statistically significant criteria was assumed at $P<0.05$ level.

\section{Results}

\section{Clinical characteristics of the study participants}

The clinical characteristics of IS patients and healthy control subjects are shown in Table 1. There were no statistically significant differences between the two groups in age, gender, hypertriglyceridemia and hypercholesterolemia $(P>0.05)$. The frequencies of abnormal LDL-cholesterol, serum total cholesterol and triglycerides, smokers, diabetes and hypertension in IS patients were significantly higher than those in the control group $(P<0.05)$. Increased levels of serum sCD40 were observed in IS patients com-

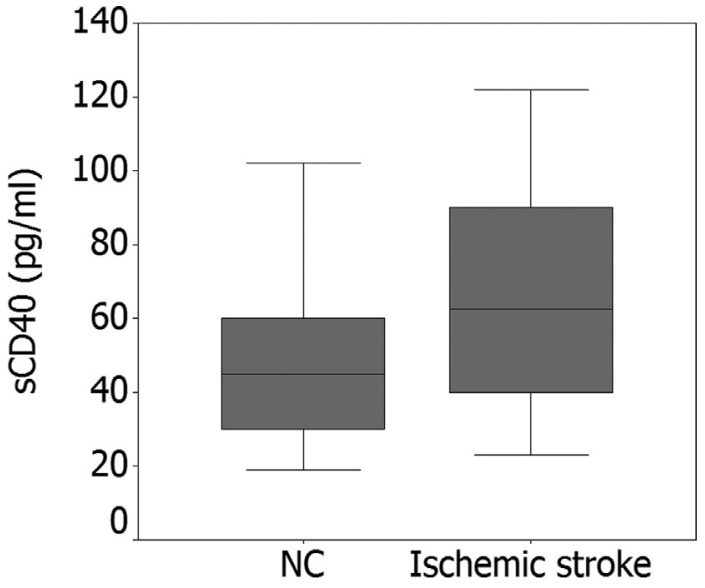

Figure 1 - The levels of sCD40 in patient with IS and the control group. Levels of sCD40 were higher in IS group than in the control group (61.3 \pm $23.7 \mathrm{pg} / \mathrm{mL}, \mathrm{n}=380 v \mathrm{vs} .44 .5 \pm 18.7 \mathrm{pg} / \mathrm{mL}, \mathrm{n}=450), P<0.001$.

pared with the control group [(61.3 $\pm 23.7 \mathrm{pg} / \mathrm{mL}, \mathrm{n}=380)$ vs $(44.5 \pm 18.7 \mathrm{pg} / \mathrm{mL}, \mathrm{n}=450) ; P<0.001]$ (Figure 1).

\section{Genotype and allele frequencies of the four polymorphisms}

All the four polymorphisms showed three genotypes according to sequencing results. The distribution of the genotype and allele frequencies of the rs1883832, rs13040307, rs752118 and rs3765459 polymorphisms in IS patients and control subjects are presented in Table 2. The genotype distribution of the four polymorphisms among IS patients and control subjects were in HWE. The frequencies of the rs $1883832 \mathrm{CC}$, rs $1883832 \mathrm{CT}$ and rs $1883832 \mathrm{TT}$ genotypes were $26.6,48.7$ and $24.7 \%$ in IS patients and $36.0,46.4$ and $17.6 \%$ in the control group, respectively. There were statistically significant differences in the genotype and allele frequencies of the rs 1883832 polymorphism between IS patients and the control group $(P<0.01)$. The

Table 1 - Clinical characteristics of the study participants.

\begin{tabular}{lccc}
\hline Variable & $\begin{array}{c}\text { Control subjects } \\
\mathrm{n}=450(\%)\end{array}$ & Stroke patients \\
$\mathrm{n}=380(\%)$ & $0.7 \pm 13.2$ & 0.102 \\
\hline Age (mean $\pm \mathrm{SD})$ & $63.9 \pm 10.3$ & $290 / 90$ & 0.180 \\
Sex (M/F) & $325 / 125$ & $212(55.8)$ & 0.002 \\
Smokers & $203(45.1)$ & $210(55.3)$ & $<0.001$ \\
Hypertension & $165(36.7)$ & $76(20.0)$ & 0.001 \\
Diabetes & $53(11.8)$ & $55(14.5)$ & 0.147 \\
Hypercholesterolemia & $50(11.1)$ & $46(12.1)$ & 0.476 \\
Hypertriglyceridemia & $62(13.8)$ & $5.29 \pm 1.22$ & 0.023 \\
Total cholesterol (mmol/L) & $4.86 \pm 1.08$ & $2.09 \pm 1.56$ & 0.001 \\
Triglycerides $(\mathrm{mmol} / \mathrm{L})$ & $1.54 \pm 0.97$ & $1.31 \pm 0.36$ & 0.038 \\
HDL-cholesterol $(\mathrm{mmol} / \mathrm{L})$ & $1.69 \pm 0.46$ & $2.98 \pm 0.93$ & 0.002 \\
LDL-cholesterol(mmol/l) & $2.31 \pm 0.98$ & & \\
\hline
\end{tabular}


Table 2 - Distribution of the genotype and allele frequencies four polymorphisms of the CD40 gene in ischemic stroke (IS) patients and control subjects.

\begin{tabular}{|c|c|c|c|c|c|}
\hline Polymorphisms & $\begin{array}{c}\text { Control subjects } \\
\mathrm{n}=450(\%)\end{array}$ & $\begin{array}{c}\text { IS patients } \mathrm{n}=380 \\
(\%)\end{array}$ & OR $(95 \% \mathrm{CI})$ & $\chi^{2}$ & $P$ value \\
\hline \multicolumn{6}{|l|}{ rs1883832 } \\
\hline $\mathrm{CC}$ & $162(36.0)$ & $101(26.6)$ & 1.00 & & \\
\hline $\mathrm{CT}$ & $209(46.4)$ & $185(48.7)$ & $1.42(1.03-1.95)$ & 4.692 & 0.030 \\
\hline TT & 79 (17.6) & 94 (24.7) & $1.91(1.29-2.82)$ & 10.175 & 0.001 \\
\hline $\mathrm{C}$ & $533(59.2)$ & $387(50.9)$ & 1.00 & & \\
\hline $\mathrm{T}$ & $367(40.8)$ & $373(49.1)$ & $1.40(1.15-1.70)$ & 11.493 & 0.001 \\
\hline \multicolumn{6}{|l|}{ rs13040307 } \\
\hline $\mathrm{CC}$ & $265(58.9)$ & $224(58.9)$ & 1.00 & & \\
\hline $\mathrm{CT}$ & $161(35.8)$ & $130(34.2)$ & $0.96(0.71-1.28)$ & 0.095 & 0.758 \\
\hline $\mathrm{TT}$ & $24(5.3)$ & $26(6.8)$ & $1.28(0.72-2.30)$ & 0.699 & 0.403 \\
\hline $\mathrm{C}$ & $691(76.8)$ & $578(76.1)$ & 1.00 & & \\
\hline $\mathrm{T}$ & $209(23.2)$ & $182(23.9)$ & $1.04(0.83-1.31)$ & 0.120 & 0.729 \\
\hline \multicolumn{6}{|l|}{ rs752118 } \\
\hline $\mathrm{CC}$ & $276(61.3)$ & $236(62.1)$ & 1.00 & & \\
\hline $\mathrm{CT}$ & $148(32.9)$ & $127(33.4)$ & $1.00(0.75-1.35)$ & 0.001 & 0.981 \\
\hline TT & $26(5.8)$ & $17(4.5)$ & $0.77(0.41-1.44)$ & 0.688 & 0.407 \\
\hline $\mathrm{C}$ & $700(77.8)$ & $599(78.8)$ & 1.00 & & \\
\hline $\mathrm{T}$ & $200(22.2)$ & $161(21.2)$ & $0.94(0.74-1.19)$ & 0.261 & 0.610 \\
\hline \multicolumn{6}{|l|}{ rs3765459 } \\
\hline GG & $249(55.3)$ & $223(58.7)$ & 1.00 & & \\
\hline GA & $171(38.0)$ & $134(35.3)$ & $0.88(0.66-1.17)$ & 0.818 & 0.366 \\
\hline AA & $30(6.7)$ & $23(6.1)$ & $0.86(0.48-1.52)$ & 0.284 & 0.594 \\
\hline G & $669(74.3)$ & $580(76.3)$ & 1.00 & & \\
\hline $\mathrm{A}$ & $231(25.7)$ & $180(23.7)$ & $0.90(0.72-1.13)$ & 0.869 & 0.351 \\
\hline
\end{tabular}

rs $1883832 \mathrm{CT}$ and $\mathrm{rs} 1883832 \mathrm{TT}$ genotypes were associated with an increased risk of IS compared with the rs $1883832 \mathrm{CC}$ genotype $(\mathrm{OR}=1.42,95 \% \mathrm{CI}: 1.03-1.95$, $\mathrm{p}=0.030$ and $\mathrm{OR}=1.91,95 \% \mathrm{CI}: 1.29-2.82, P=0.001$, respectively). Comparing with the rs $1883832 \mathrm{C}$ allele, the rs1883832T allele was associated with an increased risk of IS (OR $=1.40,95 \%$ CI: $1.15-1.70, P=0.001)$. However, there was no significant association between IS patients and the control group in the genotype and allele frequencies of the rs13040307, rs752118 and rs3765459 polymorphisms $(P>0.05)$.

\section{Genotype and allele distribution of the rs1883832 polymorphism in different populations}

Considering the importance of the CD40 rs1883832 polymorphism in the etiology of IS, we then performed a comparison of the genotype distribution of the rs 1883832 polymorphism in different populations (Table 3), and found that the genotype distribution of rs1883832 polymorphism in our current study was significantly different from HapMap-CEU, HapMap-HCB, HapMap-JPT, HapMapYRI, HapMap-ASW, HapMap-GIH, НapMap-LWK, HapMap-MKK and HapMap-TSI populations $(P<0.05)$. However, no significant difference was found when comparing with HapMap-CHB and HapMap-CHD populations $(P>0.05)$.

\section{Haplotype analysis of the four polymorphisms}

We further performed a haplotype analysis, and the possible seven haplotypes are listed in Table 4 . The results showed that the rs1883832 polymorphism was in strong linkage disequilibrium (LD) with the rs13040307 (D'= $0.873), \operatorname{rs} 752118\left(D^{\prime}=0.895\right)$ and rs3765459 $\left(D^{\prime}=0.898\right)$ polymorphisms. Also, the rs13040307 polymorphism was in strong LD with the rs752118 (D'=0.946) and rs3765459 $\left(D^{\prime}=0.937\right)$ polymorphisms. Moreover, the rs 752118 polymorphism was in strong LD with the rs3765459 (D'= 0.942) polymorphism. CCCG and TCCG were the two major haplotypes, and accounted for 27.1 and $44.1 \%$, and 30.6 and $42.0 \%$ in both IS patients and control subjects, respectively. As shown in Table 4, the TCCA haplotype was associated with an increased risk of IS compared with the control group $(\mathrm{OR}=2.10,95 \% \mathrm{CI}: 1.23-3.58, P=0.005)$.

\section{Association between CD40 polymorphisms and serum sCD40 levels}

The rs1883832 polymorphism was significantly associated with serum sCD40 levels in patients with IS. Individuals carrying the $\mathrm{r} 1883832 \mathrm{TT}(64.8 \pm 25.4 \mathrm{pg} / \mathrm{mL}, \mathrm{n}=94)$ or rs1883832CT genotype $(63.9 \pm 24.3 \mathrm{pg} / \mathrm{mL}, \mathrm{n}=185)$ showed significantly higher sCD40 levels compared with the rs $1883832 \mathrm{CC}$ genotype $(53.3 \pm 22.5 \mathrm{pg} / \mathrm{mL}, \mathrm{n}=101, P$ 
Table 3 - Comparison of the rs1883832 polymorphism in different populations.

\begin{tabular}{|c|c|c|c|c|c|c|}
\hline \multirow[b]{2}{*}{ Population } & \multirow[b]{2}{*}{ sample size } & \multicolumn{3}{|c|}{ Genotypes (\%) } & \multirow{2}{*}{$\begin{array}{c}\text { Minor allele (\%) } \\
\mathrm{T} \\
\end{array}$} & \multirow[b]{2}{*}{ Ethnicity } \\
\hline & & $\mathrm{CC}$ & $\mathrm{CT}$ & TT & & \\
\hline Our data & 450 & $162(36.0)$ & 209 (46.4) & $79(17.6)$ & $367(40.8)$ & Guangxi China \\
\hline HapMap-CEU* & 226 & $124(54.9)$ & 94 (41.6) & $8(3.5)$ & $55(24.3)$ & European \\
\hline HapMap-HCB ${ }^{*}$ & 86 & $40(46.5)$ & $44(51.2)$ & $2(2.3)$ & $24(27.9)$ & Asian \\
\hline HapMap-JPT* & 170 & $40(23.5)$ & $90(53.0)$ & $40(23.5)$ & $85(50.0)$ & Asian \\
\hline HapMap-YRI & 226 & $224(99.1)$ & $2(0.9)$ & - & $2(0.4)$ & African \\
\hline HapMap-ASW* & 98 & $94(95.9)$ & $4(4.1)$ & - & $4(2.0)$ & African \\
\hline НарМар-СНB & 82 & $40(48.8)$ & $30(36.6)$ & $12(14.6)$ & $27(32.9)$ & Asian \\
\hline HapMap-CHD & 170 & $72(42.4)$ & $68(40.0)$ & $30(17.6)$ & $64(37.6)$ & Asian \\
\hline HapMap-GIH* & 176 & $98(55.7)$ & $66(37.5)$ & $12(6.8)$ & $45(25.6)$ & Asian \\
\hline HapMap-LWK ${ }^{*}$ & 180 & $166(92.2)$ & $14(7.8)$ & - & $7(3.9)$ & Asian \\
\hline HapMap-MKK ${ }^{*}$ & 286 & $244(85.3)$ & $40(14.0)$ & $2(0.7)$ & $22(7.7)$ & African \\
\hline HapMap-TSI* & 176 & $68(38.6)$ & $104(59.1)$ & $4(2.3)$ & $56(31.8)$ & European \\
\hline
\end{tabular}

$* P<0.05$ comparing with our present data; CEU: Utah residents with northern and western European ancestry; HCB: Han Chinese in Beijing, China; JPT: Japanese in Tokyo, Japan; YRI: Yoruba in Ibadan, Nigeria. ASW: African ancestry in Southwest USA; CHB: Han Chinese in Beijing, China; CHD: Chinese in Metropolitan Denver, Colorado; GIH: Gujarati Indians in Houston, Texas; LWK: Luhya in Webuye, Kenya; MKK: Maasai in Kinyawa, Kenya; TSI: Toscans in Italy;

$<0.01)$. Nevertheless, no significant difference was found in the serum sCD40 levels between rs1883832TT and rs 1883832 CT genotypes (Figure 2). Furthermore, there was no significant association between the CD40 rs13040307, rs752118 and rs3765459 polymorphisms and serum sCD40 levels $(P>0.05)$.

\section{Clinical and biochemical values in IS patients with different CD40 genotypes}

No significant difference was found in the genotype frequencies of the CD40 rs1883832, rs13040307, rs752118 and rs3765459 polymorphisms after stratification of IS by age, smoking status, gender, and the absence or presence of hypertriglyceridemia, hypertension and hypercholesterolemia (data not shown). Moreover, no significant difference was found between different genotypes of the CD40 polymorphisms and laboratory values (TG, TC, LDL-C and HDL-C) (data not shown).

\section{Association between CD40 polymorphisms and different subtypes of IS}

We did not find any association between the CD40 polymorphisms rs1883832, rs13040307, rs752118 and rs3765459 and IS subtypes (data not shown).

\section{Discussion}

In this study, we analyzed the effect of four polymorphisms in CD40 gene on IS risk and their effect on CD40 expression in a Chinese population. Our results indicated that the rs1883832 polymorphism was associated with an increased risk of IS. The increased risk was also found in haplotype analysis. Moreover, we found that the rs1883832 polymorphism was associated with increased CD40 expression compared with the control group. The statistical power of the study was calculated to be $82 \%$ to detect the association between rs 1883832 polymorphism and IS risk in a sample size of 830 participants ( 380 in IS group and 450 in the control group) assuming an OR of 1.5 and $\alpha$ of 0.05 (NCSS PASS 11 software, version 11.0.7). Therefore, this finding indicates that the rs 1883832 polymorphism may play a crucial role in the etiology of IS.

Atherosclerosis is a major cause of cardiovascular diseases, including stroke. It is accepted that interactions between CD40 and its ligand CD40L are closely involved in the pathogenesis of inflammation, atherosclerosis and thrombosis (Anand et al., 2003, Antoniades et al., 2009). Upregulation of CD40 and/or CD40L have been detected in serum/plasma/cell surface of patients with IS by several studies, and were suggested to be useful predictors and biomarker for stroke (Cha et al., 2003; Garlichs et al., 2003; Ishikawa et al., 2005; Davi et al., 2009; Zhang et al., 2014; Li et al., 2015; Wang et al., 2015). However, the exact mechanism of how CD40 is up regulated, and how the upregulated CD40 affects IS patients have not been fully elucidated. Garlichs et al. (2003) reported that patients with acute cerebral ischemia show upregulating CD40-CD40L system expression and may contribute to a proinflammatory, proatherogenic and prothrombotic milieu. Very recently, Wang et al. (2015) investigated the association between $\mathrm{SCD} 40 \mathrm{~L}$ and carotid plaque in patients with acute ischemic stroke and found that $\mathrm{SCD} 40 \mathrm{~L}$ levels were significantly associated with carotid plaque formation and instability, suggesting that SCD40L may be a useful predictor for plaque formation and instability in patients with acute ischemic stroke. In this study, we demonstrated that serum sCD40 levels were significantly elevated in patients with IS compared with the control group. Moreover, we found that the rs1883832 polymorphism was associated with an increased serum sCD40 levels in patients with IS compared 
Table 4 - Haplotype analysis of the four polymorphism between ischemic stroke (IS) patients and control subjects.

\begin{tabular}{lcccc}
\hline $\begin{array}{l}\text { Haplotypes of CD40 polymorphisms } \\
\text { (rs1883832/rs13040307/rs752118/ rs3765459) }\end{array}$ & $\begin{array}{c}\text { Controls } \\
2 \mathrm{n}=900(\%)\end{array}$ & $\begin{array}{c}\text { IS patients } \\
2 \mathrm{n}=760(\%)\end{array}$ & OR $(95 \% \mathrm{CI})$ & $P$ value \\
\hline TCCA & $22(2.4)$ & $38(5.0)$ & $2.10(1.23-3.58)$ & 0.005 \\
CCCG & $275(30.6)$ & $206(27.1)$ & $0.84(0.68-1.05)$ & 0.123 \\
TCCG & $378(42.0)$ & $335(44.1)$ & $1.09(0.90-1.32)$ & 0.394 \\
CTTA & $190(21.1)$ & $148(19.5)$ & $0.90(0.71-1.15)$ & 0.409 \\
CTCG & $10(1.1)$ & $13(1.7)$ & $1.55(0.68-3.55)$ & 0.298 \\
CTTG & $16(1.8)$ & $11(1.4)$ & $0.81(0.37-1.76)$ & 0.596 \\
CTCA & $9(1.0)$ & $9(1.2)$ & $1.19(0.47-3.00)$ & 0.718 \\
\hline
\end{tabular}

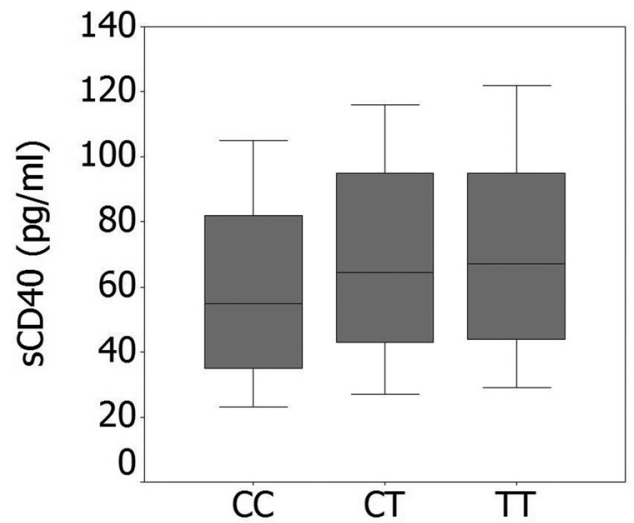

Figure 2 - Association between the CD40 rs1883832 polymorphism and sCD40 levels in IS patients. sCD40 levels were significantly lower in IS patients with rs1883832CC genotype than in rs1883832TT and rs $1883832 \mathrm{CT}$ genotypes. However, there were no significant differences in sCD40 levels between the rs1883832CT and TT genotypes.

with the control group. Genotypes carrying the rs $1883832 \mathrm{~T}$ allele were found to be associated with increased serum sCD40 levels compared with the rs $1883832 \mathrm{CC}$ genotype in patients with IS. We speculated that the rs 1883832 polymorphism may be associated with IS by up-regulating CD40 expression, which has been demonstrated to play a major role in atherosclerosis formation, plaque destabilization, thrombosis and the initiation of inflammatory response.

Several studies have been conducted to investigate the effect of the CD40 rs1883832 (-1C/T) polymorphism on atherosclerotic disease, however, the results were controversial. Yan et al. (2010) reported that the rs1883832CC genotype and the rs $1883832 \mathrm{C}$ allele in the acute coronary syndrome (ACS) group were significantly higher than those in the control group, and the rs $1883832 \mathrm{C}$ allele was found to associate with an increased risk of ACS when compared with the control group ( $\mathrm{OR}=1.88,95 \% \mathrm{CI}$ : 1.40-2.52). Similarly, a case-control study conducted by Wang et al. (2011) found that the frequency of rs $1883832 \mathrm{C}$ allele in the ACS group was significantly higher than those in the control group $(\mathrm{OR}=1.55,95 \% \mathrm{CI}: 1.15-2.11, P<$ 0.05 ), and individuals carrying the rs $1883832 \mathrm{CC}$ genotype showed a significantly increased CD40 and CD40L expression compared with the rs1883832CT and rs1883832TT genotypes carriers $(P<0.05)$. In contrast, Ma et al. (2013) reported that the frequencies of CT and TT/CT genotypes of rs1883832 polymorphism in the IS group were significantly higher than those in the control group, and the rs $1883832 \mathrm{~T}$ allele was found to be associated with a significantly increased risk of IS $(\mathrm{OR}=1.27,95 \% \mathrm{CI}=$ 1.02-1.59). Moreover, they also found that the frequency of genotypes carrying the rs $1883832 \mathrm{~T}$ allele was higher in patients with history of stroke compared with those without (for TT: $\mathrm{OR}=6.54,95 \% \mathrm{CI}=1.66-25.83$; for $\mathrm{TT} / \mathrm{CT}: \mathrm{OR}=$ $3.47,95 \%$ CI $=1.03-11.67)$. Similarly, Zhang et al. (2013) reported that the frequencies of TT genotype and T allele of the CD40 rs1883832 polymorphism were significantly higher in patients with cerebral infarction than those in the control group $(P<0.05)$. The rs 1883832TT genotype was suggested to be associated with an increased CD40 mRNA expression and CD40L plasma concentration $(P<0.01)$. Subsequently, a meta-analysis was conducted (Yun et al., 2014) and found that rs $1883832 \mathrm{C}$ allele was significantly associated with an increased risk of coronary artery disease, ACS and atherosclerosis, whereas the rs $1883832 \mathrm{C}$ allele was demonstrated to be associated with a decreased risk of IS.

Consistent with the results of Ma et al. (2013) and Zhang et al. (2013), in this study we found that the frequencies of the T allele, the TT and CT genotypes of CD40 rs1883832 polymorphism predicted a significantly higher IS risk compared with the control group (Table 2). A possible explanation for the inconsistencies between the rs1883832 polymorphism and different types of atherosclerotic diseases is that this polymorphism might have different genetic effects on different diseases. Similar results can been seen in the studies of Chen et al. (2015b) and Yi et al. (2014), in which they found that the impacts of genetic polymorphisms may be different according to the types of cancer.

Until now, very little information has been reported on the association of CD40 rs752118 and rs3765459 polymorphisms and disease susceptibility. Moreover, no data has been reported on the association between the rs13040307 polymorphism and disease susceptibility. Pre- 
vious studies conducted by Wu et al. (2016) and Wagner et al. (2015) have tried to assess the association of rs 752118 polymorphism with systemic lupus erythematosus and multiple sclerosis, respectively, but failed to obtain a positive result. Regarding the rs3765459 polymorphism, data from Shuang et al. (2011) demonstrated that the rs3765459A allele was higher in patients with breast cancer compared with the control group $(\mathrm{OR}=1.22,95 \% \mathrm{CI}$ : $1.03-1.45, P=0.025)$. However, the study conducted by Burdon et al. (2006) found that the rs3765459 polymorphism was significantly associated with a decreased risk of coronary artery calcification in diabetic families. Furthermore, rs3765459 has also been assessed in relation to systemic lupus erythematosus (Wu et al., 2016) and asthma (Hsieh et al., 2009), but a significant association was not detected.

In this study, we investigated the association between rs 13040307 , rs752118 and rs3765459 polymorphisms and risk of IS, but found no significant association. The reason for these negative results remain unknown, but two possibilities should be considered. First, it may be because of genetic trait differences, as we know that genetic polymorphisms in human genes are distinct in different ethnicities, populations and geographic regions. Data from Table 3 can support this viewpoint, as it shows that the genotype distribution of rs1883832 polymorphism in our study were significantly different from HapMap-CEU, HapMap-HCB, HapMap-JPT, HapMap-YRI, HapMap-ASW, HapMapGIH, HapMap-LWK, HapMap-MKK and HapMap-TSI populations, but similar with HapMap-CHB and HapMapCHD populations. In addition, IS is a multi-factorial disease, thus, individual exposure to diverse environmental factors and genetic background may cause different results.

Several limitations should be considered in our study. First, a relatively small sample size of the study may have limited the statistical power of the analysis. Second, as our study population was all Chinese, the results cannot be directly applied to other ethnic groups. Third, our study was designed as a hospital-based and case-control study, and the control subjects were not selected from general population, thus, we cannot exclude the possibility of selection bias. Finally, the limitation of the assay kit may also contribute to the distinction of the results.

\section{Conclusions}

After a comprehensive comparison with other studies, we found that the rs $1883832 \mathrm{~T}$ allele but not the rs $1883832 \mathrm{C}$ allele is associated with an increased risk of IS. Moreover, we demonstrated for the first time that the rs13040307, rs752118 and rs3765459 polymorphisms in CD40 gene were not associated with IS in the Chinese population. Further study with a larger sample size is needed to confirm our results, especially in different ethnic groups.

\section{Acknowledgments}

This study was supported by National Natural Science Foundation (Nos. 81560552 and 81060243) and Natural Science Foundation of Guangxi China (No. 2011GXNSFA018198).

\section{References}

Adams Jr HP, Bendixen BH, Kappelle LJ, Biller J, Love BB, Gordon DL and Marsh 3rd EE (1993) Classification of subtype of acute ischemic stroke. Definitions for use in a multicenter clinical trial. TOAST. Trial of Org 10172 in Acute Stroke Treatment. Stroke 24:35-41.

Anand SX, Viles-Gonzalez JF, Badimon JJ, Cavusoglu E and Marmur JD (2003) Membrane-associated CD40L and sCD40L in atherothrombotic disease. Thrombosis Haemostasis 90:377-384.

Antoniades C, Bakogiannis C, Tousoulis D, Antonopoulos AS and Stefanadis C (2009) The CD40/CD40 ligand system: linking inflammation with atherothrombosis. J Am Coll Cardiol 54:669-677.

Aukrust P, Damas JK and Solum NO (2004) Soluble CD40 ligand and platelets: self-perpetuating pathogenic loop in thrombosis and inflammation? J Am Coll Cardiol 43:2326-2328.

Burdon KP, Langefeld CD, Beck SR, Wagenknecht LE, Carr JJ, Rich SS, Freedman BI, Herrington D and Bowden DW (2006) Variants of the CD40 gene but not of the CD40L gene are associated with coronary artery calcification in the Diabetes Heart Study (DHS). Am Heart J 151:706-711.

Cha JK, Jeong MH, Jang JY, Bae HR, Lim YJ, Kim JS, Kim SH and Kim JW (2003) Serial measurement of surface expressions of CD63, P-selectin and CD40 ligand on platelets in atherosclerotic ischemic stroke. A possible role of CD40 ligand on platelets in atherosclerotic ischemic stroke. Cerebrovasc Dis 16:376-382.

Chen JM, Guo J, Wei CD, Wang CF, Luo HC, Wei YS and Lan Y (2015a) The association of CD40 polymorphisms with CD40 serum levels and risk of systemic lupus erythematosus. BMC Genet 16:121.

Chen P, Sun R, Pu Y, Bai P, Yuan F, Liang Y, Zhou B, Wang Y, Sun Y, Zhu J, et al. (2015b) Pri-Mir-34b/C and Tp-53 polymorphisms are associated with The susceptibility of papillary thyroid carcinoma: A case-control study. Medicine 94:e1536.

Chen Y, Chen J, Xiong Y, Da Q, Xu Y, Jiang X and Tang H (2006) Internalization of CD40 regulates its signal transduction in vascular endothelial cells. Biochem Biophys Res Commun 345:106-117.

Davi G, Tuttolomondo A, Santilli F, Basili S, Ferrante E, Di Raimondo D, Pinto A and Licata G (2009) CD40 ligand and MCP-1 as predictors of cardiovascular events in diabetic patients with stroke. J Atheroscler Thromb 16:707-713.

Elgueta R, Benson MJ, de Vries VC, Wasiuk A, Guo Y and Noelle RJ (2009) Molecular mechanism and function of CD40/CD40L engagement in the immune system. Immunol Rev 229:152-172.

Feigin VL, Lawes CM, Bennett DA, Barker-Collo SL and Parag V (2009) Worldwide stroke incidence and early case fatality reported in 56 population-based studies: A systematic review. Lancet Neurol 8:355-369. 
Garlichs CD, Kozina S, Fateh-Moghadam S, Handschu R, Tomandl B, Stumpf C, Eskafi S, Raaz D, Schmeisser A, Yilmaz A, et al. (2003) Upregulation of CD40-CD40 ligand (CD154) in patients with acute cerebral ischemia. Stroke 34:1412-1418.

Hsieh YY, Wan L, Chang CC, Tsai CH and Tsai FJ (2009) STAT $2 *$ C related genotypes and allele but not TLR4 and CD40 gene polymorphisms are associated with higher susceptibility for asthma. Int J Biol Sci 5:74-81.

Ishikawa M, Vowinkel T, Stokes KY, Arumugam TV, Yilmaz G, Nanda A and Granger DN (2005) CD40/CD40 ligand signaling in mouse cerebral microvasculature after focal ischemia/reperfusion. Circulation 111:1690-1696.

Jacobson EM, Concepcion E, Oashi T and Tomer Y (2005) A Graves' disease-associated Kozak sequence single-nucleotide polymorphism enhances the efficiency of CD40 gene translation: a case for translational pathophysiology. Endocrinology 146:2684-2691.

John SW, Weitzner G, Rozen R and Scriver CR (1991) A rapid procedure for extracting genomic DNA from leukocytes. Nucleic Acids Res 19:408.

Li J, Wang Y, Lin J, Wang D, Wang A, Zhao X, Liu L, Wang C and Wang Y (2015) Soluble CD40L is a useful marker to predict future strokes in patients with minor stroke and transient ischemic attack. Stroke 46:1990-1992.

Liu L, Wang D, Wong KS and Wang Y (2011) Stroke and stroke care in China: huge burden, significant workload, and a national priority. Stroke 42:3651-3654.

Lloyd-Jones D, Adams RJ, Brown TM, Carnethon M, Dai S, De Simone G, Ferguson TB, Ford E, Furie K, Gillespie C, et al. (2010) Heart disease and stroke statistics - 2010 update: a report from the American Heart Association. Circulation 121:e46-e215.

Ma Y, Wang SX, Liu Y, Peng GG, Wang XM, Zhang B, Wu BH and Yu JM (2013) Single nucleotide polymorphism of CD40 in the 5'-untranslated region is associated with ischemic stroke. Gene 529:257-261.

Sacco RL, Benjamin EJ, Broderick JP, Dyken M, Easton JD, Feinberg WM, Goldstein LB, Gorelick PB, Howard G, Kittner SJ, et al. (1997) American Heart Association Prevention Conference. IV. Prevention and rehabilitation of stroke. Risk factors. Stroke 28:1507-1517.

Sacco RL, Ellenberg JH, Mohr JP, Tatemichi TK, Hier DB, Price TR and Wolf PA (1989) Infarcts of undetermined cause: The NINCDS Stroke Data Bank. Ann Neurol 25:382-390.

Schonbeck U, Gerdes N, Varo N, Reynolds RS, Horton DB, Bavendiek U, Robbie L, Ganz P, Kinlay S and Libby P (2002) Oxidized low-density lipoprotein augments and 3hydroxy-3-methylglutaryl coenzyme A reductase inhibitors limit CD40 and CD40L expression in human vascular cells. Circulation 106:2888-2893.

Schonbeck U and Libby P (2001) CD40 signaling and plaque instability. Circulation Res 89:1092-1103.

Shi YY and He L (2005) SHEsis, a powerful software platform for analyses of linkage disequilibrium, haplotype construction, and genetic association at polymorphism loci. Cell Res 15:97-98.
Shuang C, Dalin L, Weiguang Y, Zhenkun F, Fengyan X, Da P and Li D (2011) Association of CD40 gene polymorphisms with sporadic breast cancer in Chinese Han women of Northeast China. PloS One 6:e23762.

Stephens M, Smith NJ and Donnelly P (2001) A new statistical method for haplotype reconstruction from population data. Am J Hum Genet 68:978-989.

Tian C, Qin W, Li L, Zheng W and Qiu F (2010) A common polymorphism in CD40 Kozak sequence $(-1 \mathrm{C} / \mathrm{T})$ is associated with acute coronary syndrome. Biomed Pharmacother 64:191-194.

Turtzo LC and McCullough LD (2010) Sex-specific responses to stroke. Future Neurol 5:47-59.

Wagner M, Sobczynski M, Bilinska M, Pokryszko-Dragan A, Cyrul M, Kusnierczyk P and Jasek M (2015) MS risk allele rs $1883832 \mathrm{~T}$ is associated with decreased mRNA expression of CD40. J Mol Neurosci 56:540-545.

Wang M, Li Y, Li W, Xia ZE and Wu Q (2011) The CD40 gene polymorphism rs1883832 is associated with risk of acute coronary syndrome in a Chinese case-control study. DNA Cell Biol 30:173-178.

Wang YJ, Gong ZQ, Bi XM and Li YL (2015) Correlation of plasma soluble cluster of differentiation 40 ligand, alpha fetoprotein A, and pregnancy-associated plasma protein A with carotid plaque in patients with ischemic stroke. Genet Mol Res 1:8091-8099.

Wu CJ, Guo J, Luo HC, Wei CD, Wang CF, Lan Y and Wei YS (2016) Association of CD40 polymorphisms and haplotype with risk of systemic lupus erythematosus. Rheumatol Int 36:45-52.

Yan J, Wang C, Du R, Liu P and Chen G (2010) Association analysis of CD40 gene polymorphism with acute coronary syndrome. Clin Exp Med 10:253-258.

Yan J, Wu Z, Huang Z, Li L, Zhong R and Kong X (2002) Clinical implications of increased expression of CD40L in patients with acute coronary syndromes. Chin Med J 115:491-493.

Yan JC, Zhu J, Gao L, Wu ZG, Kong XT, Zong RQ and Zhan LZ (2004) The effect of elevated serum soluble CD40 ligand on the prognostic value in patients with acute coronary syndromes. Clin Chim Acta 343:155-159.

Yi DH, Wang BG, Zhong XP, Liu H and Liu YF (2014) PrimiR-34b/c rs4938723 TC heterozygote is associated with increased cancer risks: evidence from published data. Tumour Biol 35:11967-11975.

Yun Y, Ma C and Ma X (2014) The SNP rs1883832 in CD40 gene and risk of atherosclerosis in Chinese population: A metaanalysis. PLoS One 9:e97289.

Zhang B, Chen M, Yang H, Wu T, Song C and Guo R (2014) Evidence for involvement of the CD40/CD40L system in poststroke epilepsy. Neurosci Lett 567:6-10.

Zhang B, Wu T, Song C, Chen M, Li H and Guo R (2013) Association of $\mathrm{CD} 40-1 \mathrm{C} / \mathrm{T}$ polymorphism with cerebral infarction susceptibility and its effect on SCD40L in Chinese population. Int Immunopharmacol 16:461-465.

\section{Associate Editor: Mara H. Hutz}

License information: This is an open-access article distributed under the terms of the Creative Commons Attribution License (type CC-BY), which permits unrestricted use, distribution and reproduction in any medium, provided the original article is properly cited. 